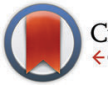

CrossMark \& click for updates

Cite this: Chem. Commun., 2016, 52,14027

Received 10th October 2016 Accepted 28th October 2016

DOI: $10.1039 / c 6 c c 08164 a$

www.rsc.org/chemcomm

\section{Enzymatic synthesis of natural (+)-aristolochene from a non-natural substrate $\dagger$}

\author{
Juan A. Faraldos, ${ }^{a}$ Daniel J. Grundy, ${ }^{a}$ Oscar Cascon, ${ }^{a}$ Stefano Leoni, ${ }^{a}$ \\ Marc W. van der Kamp ${ }^{b}$ and Rudolf K. Allemann*a
}

The sesquiterpene cyclase aristolochene synthase from Penicillium roquefortii (PR-AS) has evolved to catalyse with high specificity (92\%) the conversion of farnesyl diphosphate (FDP) to the bicyclic hydrocarbon $(+)$-aristolochene, the natural precursor of several fungal toxins. Here we report that PR-AS converts the unnatural FDP isomer 7-methylene farnesyl diphosphate to (+)-aristolochene via the intermediate 7-methylene germacrene A. Within the confined space of the enzyme's active site, PR-AS stabilises the reactive conformers of germacrene $A$ and 7-methylene germacrene $A$, respectively, which are protonated by the same active site acid (most likely HOPP) to yield the shared natural bicyclic intermediate eudesmane cation, from which (+)-aristolochene is then generated.

Terpenoids are found in all forms of life and comprise one of the largest and most structurally diverse families of natural products. ${ }^{1-4}$ Sesquiterpenoids are biosynthesized from the universal linear C15-precursor farnesyl diphosphate (FDP, 1), which is converted in electrophilic cyclization reactions of often exquisite specificity to $>300$ mostly cyclic hydrocarbon products in class I sesquiterpene synthase catalysed processes. ${ }^{5}$ The electrophilic nature and stereochemical precision of these cascade reactions have been probed in vitro with FDP isotopologues ${ }^{6-11}$ as well as with substituted FDPs that carry fluoro, ${ }^{12-16}$ methyl ${ }^{17,18}$ and phenyl substituents. ${ }^{19}$ The ability of sesquiterpene synthases to bind such synthetic FDP analogues as competitive inhibitors ${ }^{13,19,20}$ and occasionally as substrates ${ }^{11,12,14,16-18}$ demonstrates their plasticity and catalytic flexibility, properties that have been exploited for the synthesis of several complex unnatural molecules. . $^{12,14,17,21}$

Aristolochene synthase from Penicillium roquefortii (PR-AS) efficiently (92\%) catalyses the cyclisation of 1 to the bicyclic

\footnotetext{
${ }^{a}$ School of Chemistry, Cardiff University, Park Place, Cardiff CF10 3AT, UK. E-mail: AllemannRK@cardiff.ac.uk

${ }^{b}$ School of Biochemistry, Faculty of Biomedical Sciences \& Centre for Computational Chemistry, School of Chemistry, University of Bristol, Biomedical Sciences Building, University Walk, Bristol BS8 1TD, UK $\dagger$ Electronic supplementary information (ESI) available: Synthesis of FDP and 7-methylene-FDP, gas chromatograms, MS spectra, ${ }^{1} \mathrm{H}-\mathrm{NMR}$ spectra and molecular calculations. See DOI: 10.1039/c6cc08164a
}

eremophilane hydrocarbon (+)-aristolochene (2), the precursor of several highly oxygenated mycotoxins such us PR-toxin. ${ }^{8,22}$ This cyclisation, which generates two 6-membered rings and introduces two double bonds and three stereo-centres with high specificity, ${ }^{23-25}$ goes through the neutral intermediate germacrene A (3), a flexible cyclodecadiene hydrocarbon that undergoes reprotonation to eudesmane cation (4), ${ }^{24,25}$ most likely by proton transfer from the by-product diphosphate $\left(\mathrm{HOPP}_{\mathrm{i}}\right)^{26-28}$ Rearrangement of $\mathbf{4}$ by way of a hydride and a methyl shift is followed by a selective deprotonation at $\mathrm{C} 8$ to yield (+)-aristolochene (2) (Scheme 1) together with a small amount of $\sim 1 \%$ valencene (5), the double bond isomer of 2 that results from deprotonation at C6 rather than C8. PR-AS also releases $\sim 7 \%$ germacrene A (3) prior to protonation of the central C6,C7-double bond of 3, possibly as a consequence of a conformational change prior to eudesmane cation formation. ${ }^{24,25}$
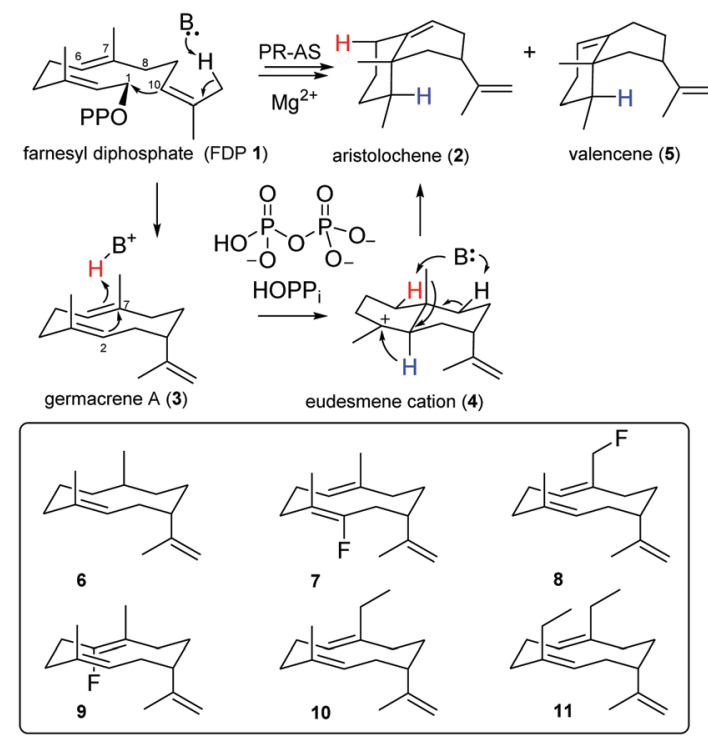

Scheme 1 Biosynthesis of (+)-aristolochene (2) and unnatural germacrenoids (6-11) generated by PR-AS from the corresponding FDP analogues. 
The intermediacy of eudesmane cation (4) was inferred from site directed mutagenesis experiments ${ }^{29,30}$ and inhibition studies with ammonium and iminium salt analogues of $4 .^{31,32}$ Evidence for the formation of germacrene A (3) was first obtained when PR-AS was challenged with 6,7-dihydroFDP, which was turned over to the corresponding dihydrogermacrene A (6). ${ }^{21}$ Similarly, the PR-AS catalysed turnover of several fluoro- and methyl-substituted FDP analogues to the corresponding altered germacrene A analogues $(6-11)^{12,14,17,21}$ illustrated the synthetic potential of sesquiterpene synthases for organic synthesis. ${ }^{11,14,16-18}$

If $\mathrm{HOPP}_{\mathrm{i}}$ protonates germacrene A (3) and its corresponding base ( ${ }^{-} \mathrm{OPPi}$ ) acts to abstract a proton from eudesmane cation (4) in the final step of PR-AS catalysis ${ }^{27,28}$ (Scheme 1), the synthetic space accessible to PR-AS might potentially be expandable and 7-methylene FDP (12), an unnatural FDP isomer that the enzyme will never be exposed to in the host organism, could be efficiently converted to aristolochene by reprotonation of the $\mathrm{C} 7, \mathrm{C} 14$ double bond of the intermediate 7-methylene germacrene A (13) to yield eudesmane cation (4) (Scheme 2), accomplishing for the first time a one-step enzymatic synthesis of a structurally complex natural sesquiterpene (2) from a non-natural, synthetic precursor. Comparison of the relative reaction rates of the hydration for a series of simple olefins in aqueous sulfuric acid ${ }^{33}$ suggests that both 3 and 13 should have similar reactivity towards protonation under general acid catalysis.

To assess the feasibility of the PR-AS catalysed cyclisation of 7-methylene-FDP (12) to 2, quantum mechanical geometry optimisation and docking into the enzyme active were performed. Previously, it was suggested that 3 exists as a mixture of three conformers in solution with the UU conformer (enforced by PR-AS, Fig. 1) as the predominant form (52\%). ${ }^{34}$ Molecular modeling, in which the hydrocarbon chain was confined to the approximate active site volume, confirmed that like for $\mathbf{3}$, the most stable conformation of $\mathbf{1 3}$ is the $\mathrm{UU}$ form. Upon relaxation in vacuo, distances between $\mathrm{C} 2$ and $\mathrm{C} 7$ in 3 and 13 are $\sim 3.3 \AA$. When the volume accessible to 3 and $\mathbf{1 3}$ is constrained, marked shortening of the $\mathrm{C} 2-\mathrm{C} 7$ distances were observed to less than $2.5 \AA$ (Fig. 1). Docking of the constrained structures of 3 and 13 in the (highly homologous) closed active site of aristolochene synthase from A. terreus $^{35}$ (Fig. 2) demonstrates that both fit in the active site pocket in similar conformations and in an

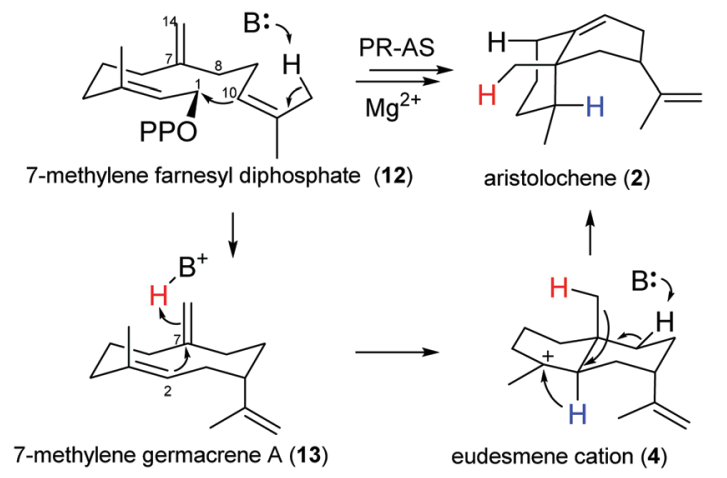

Scheme 2 The PR-AS catalysed conversion of 7-methylene-FDP (12) to (+)-aristolochene (2).
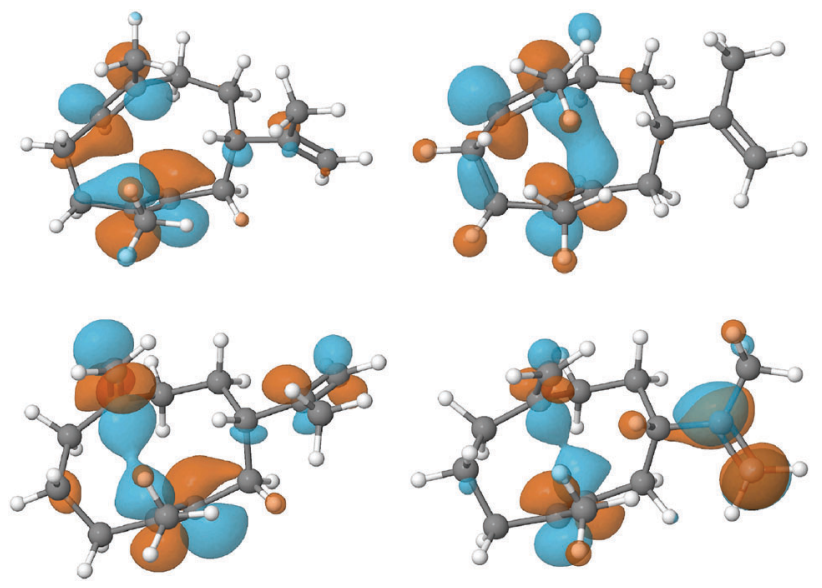

Fig. 1 Geometries and LUMOs of $\mathbf{3}$ (above) and $\mathbf{1 3}$ (below) in their reactive UU conformations, after unconstrained optimisation (vacuum) (left) and constrained optimisation and docking into the enzyme active site (right). Under constrained volume conditions 3 and $\mathbf{1 3}$ become more similar and the 2,7-contact shorter than the 2,6 contact (see also Fig. S2, ESI $\dagger$ ).

orientation consistent with the expected reaction path, with e.g. - OPPi abstracting a proton from 1 or 12. As an indicator of the direction of the second ring closure reaction (Scheme 1), we modeled the LUMOs of $\mathbf{3}$ and $\mathbf{1 3}$ both in vacuo and in the enzyme environment. Upon constrained optimisation of the vacuum relaxed structures, the nodal configurations markedly changed for both 3 and 13, and they remain similar when the enzyme environment is taken into account. The calculated LUMOs highlight the favourable orbital overlap across the ring and demonstrate the feasibility of the acid-catalysed C2,C7 ring closure for both 3 and 13 inside PR-AS (Fig. 1).

After expressing the PR-AS cDNA in E. coli (ESI $\dagger$ ), GC-MS analysis of incubations of 7-methylene FDP (12) with purified recombinant PR-AS revealed the in vitro formation of a $5: 3$ mixture of two major products (Fig. 3). Comparison of the elution profiles in the gas chromatograms and MS fragmentation patterns from parallel incubations of $\mathbf{1}$ and $\mathbf{1 2}$ with PR-AS and Solidago canadensis germacrene A synthase $(\mathrm{GAS})^{36}$ indicated that the

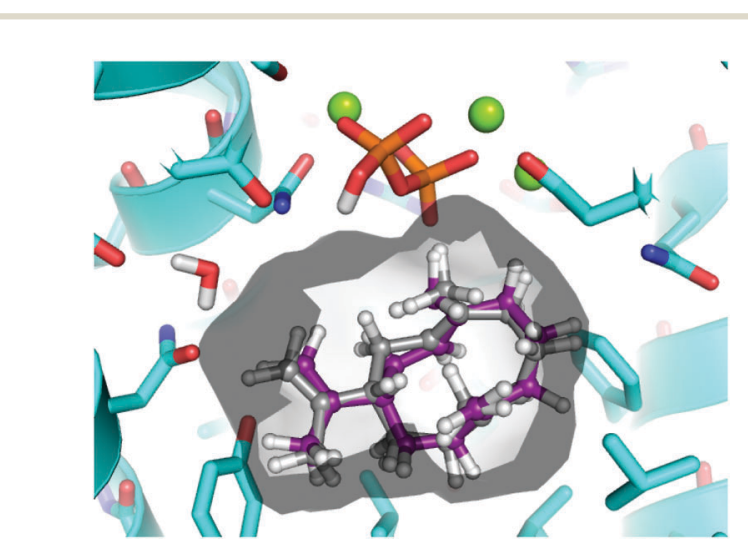

Fig. 2 Constrained geometries of $\mathbf{3}$ (grey carbons) and $\mathbf{1 3}$ (purple carbons) as docked in the active site of aristolochene synthase from $A$. terreus (cyan carbons; PDB ID: 4KVY, chain A). The solvent accessible surface of the enzyme active site is shown. For clarity, hydrogen atoms are removed from protein residues. 


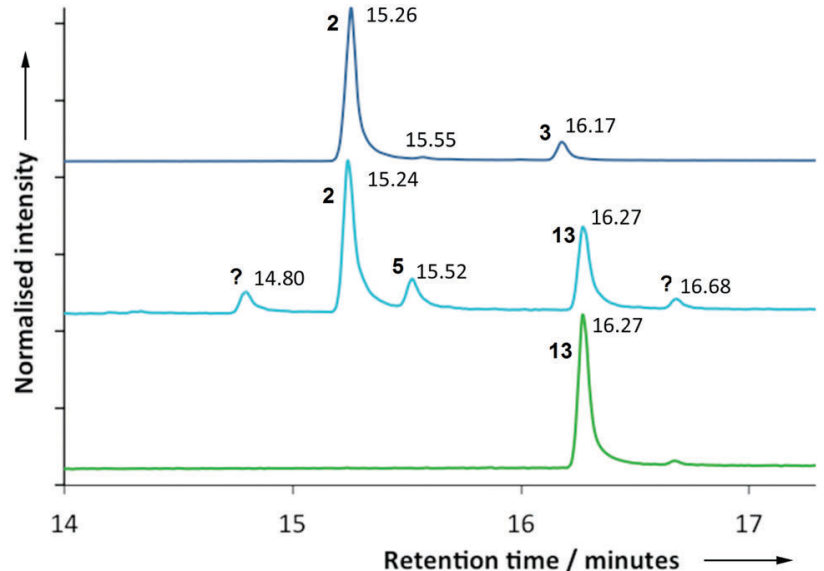

Fig. 3 Gas chromatographic analyses of the products produced from incubations of PR-AS with FDP (1) (blue, top) and 7-methylene FDP (12) (light blue, middle). For comparison, the result from an incubation of germacrene $A$ synthase (GAS) with 7-methylene FDP (12) is also shown (green, bottom).

two products generated by PR-AS from 12 were aristolochene (2) (50.1\%, 15.24 minutes) and the germacrene A analogue 13 (30.1\%, 16.27 minutes). In addition to 2 and 13 three minor products were formed (Fig. 3) that made up $19.8 \%$ of the total amount of products. Their identity was not unambiguously determined but the possibility that they were linear 7-methylene farnesenes was ruled out by comparison of the GC chromatograms and MS spectra of the products obtained from the reaction of 12 with Mentha X piperita (E)- $\beta$-farnesene synthase (EBFS). ${ }^{37}$ The GC retention times and comparison with the MS of the products formed from incubations of 1 with PR-AS suggested that the compound that eluted at 15.55 (ca. 10\%) min was valencene (5) (Fig. 3). The production of 2 from diphosphate 12 suggests that both reactions go via eudesmane cation (4) (Schemes 1 and 2). In the case of $\mathbf{1 3}$, the exo double bond may be positioned slightly less favourably for protonation so that $\mathbf{1 3}$ is longer lived in the active site than $\mathbf{3}$ and more germacrenoid $\mathbf{1 3}$ $(30 \%)$ is released than is the case for $\mathbf{3}(7 \%)$. Alternatively, 13 might bind less tightly to the active site of PR-AS. Nevertheless, the similarity of $\mathbf{1}$ and $\mathbf{1 2}$ and the plasticity of PR-AS lead to similar active site conformations for both substrates so that the initial 1,10-ring closure reactions to 3 and 13 occur along closely related reaction paths. The molecular modeling results demonstrate the importance of constraining the germacrene A intermediates $\mathbf{3}$ and $\mathbf{1 3}$ in the active site for the protonation of the endo-C6,C7 and exo-C7,C14 double bonds.

The investigation reported here demonstrates the remarkable versatility of PR-AS, which effectively turns over an unnatural synthetic precursor to the natural products aristolochene (2) and valencene (5). The PR-AS catalysed conversion of the unnatural substrate 12 to (+)-aristolochene (2) illustrates the power of substrate engineering for the in vitro synthesis of structurally complex natural products utilizing sesquiterpene synthases as biocatalysts. Our in silico work also highlights the importance of constraining the reactants for the reactivity of intermediates towards the generation of complex terpene products, an effect that has first been seen in the relatively easy, albeit less specific biomimetic cyclisation reactions of squalene in the absence of enzyme, where the hydrophobic effect leads to restrictions of the conformational space accessible to the hydrocarbon chain. ${ }^{38}$ For shorter chain isoprenoid diphosphates such as geranyl diphosphate, farnesyl diphosphate and geranylgeranyl diphosphate on the other hand, the enzyme is needed to act as a folding template for efficient product formation.

This work was supported by the UK's Biotechnology and Biological Sciences Research Council (BB/H01683X/1, BB/G003572/1 and BB/M026280/1), the UK's Engineering and Physical Sciences Research Council (EP/L027240/1) and the Cardiff Synthetic Biology Initiative, Cardiff University. We thank Dr Rob Jenkins, Robin Hicks, Simon Waller and Thomas Williams (Cardiff University) for assistance with mass spectrometry and NMR, and Dr Veronica Gonzalez (Cardiff University) for the preparation of GAS and EBFS. Computational resources of ARCCA at Cardiff are acknowledged.

\section{Notes and references}

1 J. Gershenzon and N. Dudareva, Nat. Chem. Biol., 2007, 3, 408.

2 J. D. Connolly and R. A. Hill, Dictionary of Terpenoids, Chapman and Hall, London, 1991.

3 D. E. Cane, Chem. Rev., 1990, 90, 1089.

4 D. W. Christianson, Chem. Rev., 2006, 106, 3412.

5 D. J. Miller and R. K. Allemann, Nat. Prod. Rep., 2012, 29, 60.

6 D. E. Cane, S. Swanson and P. P. N. Murthy, J. Am. Chem. Soc., 1981, 103, 2136.

7 D. E. Cane, H.-J. Ha, C. Pargellis, F. Waldmeier, S. Swanson and P. P. N. Murthy, Bioorg. Chem., 1985, 13, 246.

8 D. E. Cane, P. C. Prabhakaran, E. J. Salaski, P. H. M. Harrison, H. Noguchi and B. J. Rawlings, J. Am. Chem. Soc., 1989, 111, 8914.

9 C. O. Schmidt, H. J. Bouwmeester, S. Franke and W. A. König, Chirality, 1999, 362, 353.

10 S. Picaud, P. Mercke, X. He, O. Sterner, M. Brodelius, D. E. Cane and P. E. Brodelius, Arch. Biochem. Biophys., 2006, 448, 150.

11 J. A. Faraldos, D. J. Miller, V. Gonzalez, Z. Yoosuf-Aly, O. Cascon, A. Li and R. K. Allemann, J. Am. Chem. Soc., 2012, 134, 5900.

12 D. J. Miller, F. L. Yu, D. W. Knight and R. K. Allemann, Org. Biomol. Chem., 2009, 7, 962.

13 F. Yu, D. J. Miller and R. K. Allemann, Chem. Commun., 2007, 4155.

14 D. J. Miller, F. L. Yu and R. K. Allemann, ChemBioChem, 2007, 8, 1819.

15 J. P. Noel, N. Dellas, J. A. Faraldos, M. Zhao, B. A. Hess Jr., L. Smentek, R. M. Coates and P. E. O'Maille, ACS Chem. Biol., 2010, 5, 377.

16 J. A. Faraldos, Y. Zhao, P. E. O'Maille, J. P. Noel and R. M. Coates, ChemBioChem, 2007, 8, 1826.

17 O. Cascón, S. Touchet, D. J. Miller, V. Gonzalez, J. A. Faraldos and R. K. Allemann, Chem. Commun., 2012, 48, 9702.

18 S. Touchet, K. Chamberlain, C. M. Woodcock, D. J. Miller, M. A. Birkett, J. A. Pickett and R. K. Allemann, Chem. Commun., 2015, 51,7550 .

19 D. J. Miller, F. L. Yu, N. J. Young and R. K. Allemann, Org. Biomol. Chem., 2007, 5, 3287.

20 D. E. Cane and C. Bryant, J. Am. Chem. Soc., 1994, 116, 12063.

21 D. E. Cane and Y. S. Tsantrizos, J. Am. Chem. Soc., 1996, 118, 10037.

22 D. E. Cane, Z. Wu, R. H. Proctor and T. M. Hohn, Arch. Biochem. Biophys., 1993, 304, 415.

23 D. E. Cane, P. C. Prabhakaran, J. S. Oliver and D. B. McIlwaine, J. Am. Chem. Soc., 1990, 112, 3209.

24 M. J. Calvert, P. R. Ashton and R. K. Allemann, J. Am. Chem. Soc., 2002, 124, 11636.

25 B. Felicetti and D. Cane, J. Am. Chem. Soc., 2004, 126, 7212.

26 E. Shishova, L. Di Costanzo, D. E. Cane and D. W. Christianson, Biochemistry, 2007, 46, 1941.

27 J. A. Faraldos, V. Gonzalez and R. K. Allemann, Chem. Commun., 2012, 48, 3230.

28 T. A. Pemberton and D. W. Christianson, J. Antibiot., 2016, 69, 486. 
29 A. Deligeorgopoulou, S. E. Taylor, S. Forcat and R. K. Allemann, Chem. Commun., 2003, 2162.

30 J. A. Faraldos, A. K. Antonczak, V. González, R. Fullerton, E. M. Tippmann and R. K. Allemann, J. Am. Chem. Soc., 2011, 133, 13906.

31 J. A. Faraldos, B. Kariuki and R. K. Allemann, J. Org. Chem., 2010, $75,1119$.

32 J. A. Faraldos and R. K. Allemann, Org. Lett., 2011, 13, 1202-1205.

33 V. J. Nowlan and T. T. Tidwell, Acc. Chem. Res., 1977, 10, 252.

34 J. A. Faraldos, S. Wu, J. Chappell and R. M. Coates, Tetrahedron, 2007, 63, 7733.
35 M. Chen, N. Al-lami, M. Janvier, E. L. D’Antonio, J. A. Faraldos, D. E. Cane, R. K. Allemann and D. W. Christianson, Biochemistry, 2013, 52, 5441.

36 V. Gonzalez, S. Touchet, D. J. Grundy, J. A. Faraldos and R. K. Allemann, J. Am. Chem. Soc., 2014, 136, 14505.

37 J. A. Faraldos, V. Gonzalez, A. Li, F. Yu, M. Köksal, D. W. Christianson and R. K. Allemann, J. Am. Chem. Soc., 2012, 134, 20844.

38 T. D. Zahn, M. Eilers, Z. Guo, M. B. Ksebati, M. Simon, J. D. Scholten, S. O. Smith and R. A. Gibbs, J. Am. Chem. Soc., 2000, 122, 7135. 